\title{
Characterization of morphologically benign biologically aggressive meningiomas
}

\author{
Shalinee Rao, N. Sadiya, Saraswathi Doraiswami, D. Prathiba \\ Department of Pathology, Sri Ramachandra Medical College and Research Institute, Porur, Chennai - 600 116, India
}

\author{
Address for correspondence: \\ Dr. D. Prathiba, \\ Department of Pathology, \\ Sri Ramachandra Medical College \\ and Research Institute, Porur, \\ Chennai - 600 116, India. \\ E-mail: prathib@rediffmail.com
}

DOI: $10.4103 / 0028-3886.59470$

\begin{abstract}
Background: Meningiomas are presently categorized into three grades by World Health Organization (WHO). Grade I, in general, is expected to behave in a benign fashion. However, a borderline group of grade I meningioma also exists, which may behave aggressively. Aims and Objective: To evaluate the proliferation index and p53 antigen expression of meningiomas and correlate with histological grade and clinical course. Materials and Methods: All 123 cases of meningiomas, diagnosed between January 2000 and August 2007, were regraded according to WHO 2000 criteria. Immunostaining for Ki-67 antigen and p53 protein were performed on 68 cases selected for the study. Results: Six of the 68 cases presented with recurrence. Mean Ki-67 labeling index (Ki$67 \mathrm{LI}$ ) was $3.8 \%, 13.7 \%, 19.4 \%$ for grade I, II, and III cases, respectively. Multivariate analysis of mean Ki-67 LI showed statistically significant difference between subgroups. Mean p53 expression was found to be $15.5 \%, 57 \%, 60.8 \%$, and $62.5 \%$ for grade I, II, III, and recurrent cases, respectively. Multivariate analysis of mean p 53 expression showed no statistically significant difference between higher grades. All recurrent cases were histologically $\mathrm{WHO}$ grade I, and showed a high Ki-67 LI and p53 expression with mean Ki-67 LI and p53 expression of $8.6 \%$ and $62.5 \%$ respectively. Conclusion: Utilization of markers for proliferation and cell cycle regulators in combination with histopathological features helps in the identification of a subset of biologically aggressive morphologically benign meningiomas.
\end{abstract}

Key words: Meningioma, p53, Ki-67 labeling index

\section{Introduction}

Meningiomas composed of neoplastic meningothelial cells are in general indolent and slow growing tumors. They account for $13-26 \%$ of all primary intracranial tumors. ${ }^{[1]}$ Based on histological parameters, World Health Organization (WHO) presently categorizes meningiomas into three grades conveying prognosis. Histological features associated with aggressive behavior include: Loss of cytoarchitecture, increased cellularity, high mitotic activity, presence of necrosis, nuclear pleomorphism and invasion of brain parenchyma! ${ }^{[2,3]}$ Clinical features associated with tumor recurrence include: Age less than 40 years, male gender, and unfavourable location with incomplete resection.
WHO grade I meningiomas are expected to have a benign couse. However, a borderline group of grade I meningioma also exists which behaves aggressively. ${ }^{[4]}$ More specific criteria or adjunctive diagnostic techniques are required to identify these deceptively benign meningiomas. Studying the cell proliferation index and expression of gene regulating cell cycles may provide an objective method for assessing the tumor biology. $\mathrm{Ki}-67$ antigen is a nonhistone protein expressed only in proliferative phase of cell cycle (G1, S, G2 and M phases). It is considered to be the most reliable proliferative marker predicting tumor behavior and can be detected on formalin fixed paraffin embedded tissue sections. ${ }^{[5,6]}$ Multiple factors play a role in tumor progression and one such factor is mutation of tumor suppressor gene. Wild 
type gene product functions as a transcription factor and is involved in DNA repair regulation of cell cycle and induction of apoptosis. Mutated p53 lacks these properties leading to genomic instability and is a frequent target for genetic abnormalities in most tumors. ${ }^{[7]}$ Mutation of p53 gene results in metabolically stable abnormal protein that accumulates in the nucleus reaching the level to be easily detected by immunostains. ${ }^{[7]}$ Hence, expression of p53 protein on tissue sections is an indicator of possible mutation in p53 gene itself. The present study was done to assess the biological behavior of meningiomas by evaluating proliferation index and p53 antigen expression and correlate with histological grade and clinical course.

\section{Materials and Methods}

Hematoxylin and eosin ( $\mathrm{H}$ and $\mathrm{E})$ stained slides of all meningiomas diagnosed between January 2000 to August 2007 were reviewed and regraded according to WHO 2000 criteria. $^{[1]}$ Sixty-eight cases of the 123 meningiomas diagnosed during the study period were selected for the study [Table 1]. Since the incidence of grade II and grade III meningiomas was low as compared to grade I meningiomas, we included all cases of grade II and III for which paraffin sections were available [Table 1]. All recurrent cases diagnosed during the study period were included in the study. Immunolocalization was performed using supersensitive polymer-horseradish peroxidase (HRP) detection system (A biotin free detection system) for Ki-67 antigen and p53 protein, on $4 \mu$ thick paraffin embedded sections in the study cases. Immunohistochemical reactions were performed using prediluted monoclonal mouse antihuman antibody to Ki-67 antigen (Clone: BGX297; Isotype: IgG1 kappa, BioGenex) and p53 protein (Clone: DO7; Isotype: IgG2b, BioGenex). Sections were examined under high power(40X) field to observe immunoreactivity. A hot spot (area with highest density of immunostained nuclei) was selected and adjacent cells counted to include 1,000 nuclei.

Ki-67 labeling index (Ki-67 LI) was recorded as percentage of positively stained tumor nuclei in 1,000 tumor cells.

Table 1: Total number of cases reviewed and cases selected for the study

\begin{tabular}{l|c|c|c}
\hline Total cases reviewed & Grade I & Grade II & Grade III \\
\hline 123 & 108 & 10 & 5 \\
Study cases & Grade I & Grade II & Grade III \\
68 & 56 & 7 & 5 \\
\hline
\end{tabular}

Table 2: Ki-67 Ll in each grade of meningiomas

\begin{tabular}{lcc}
\hline Grade & Range in \% & Mean in \% \\
\hline I & $1-18$ & 3.8 \\
II & $12-17$ & 13.7 \\
III & $15-25$ & 19.4 \\
Recurrent cases & $7-12$ & 8.6 \\
\hline
\end{tabular}

Section of lymph node was used as positive control for Ki-67. Expression of p53 protein was identified as nuclear staining in 1,000 tumor cells and percentage calculated. Section of invasive ductal carcinoma of breast was used as positive control for p53. Mean Ki-67 LI and p53 protein expression values derived for grade I, II, III and recurrent meningiomas were the variables used for a multivariate analysis.

\section{Results}

Age at presentation ranged between 27 and 77 years and the common age group for all grades of meningioma was 41-60 years. The mean age at presentation for grade I, II, III and recurrent cases was 52.9, 47.3, 54.4, and 55.4 years respectively. There was female gender predominance, with a female to male ratio of 1.4: $1(p=0.007)$. Of the 68 cases six cases had recurrence. Mean Ki-67 LI was $3.8 \%, 13.7 \%, 19.4 \%$ and $8.6 \%$ for grade I [Figure 1], II, III [Figure 2], and recurrent cases respectively [Table 2]. The difference in mean Ki-67 LI was statistically significant on multivariate analysis [Table 3]. Some of the meningiomas showed high Ki-67 LI at focal areas of the tumor only. Mean p53 expression was found to be $15.5 \%, 57 \%, 60.8 \%$ and $62.5 \%$ for grade I [Figure 1], II, III [Figure 2] and recurrent cases respectively [Table 4]. p53 expression in WHO grade II and III showed markedly high but overlapping values. Multivariate analysis of p53 expression showed no statistical significance between the higher grades.
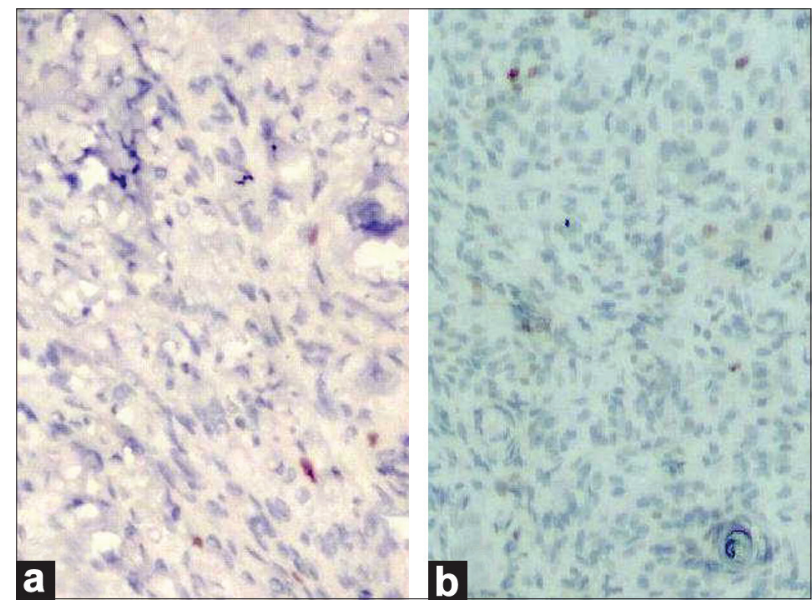

Figure 1: Sections of meningiotheliomatous meningioma-a: Occasional cells showing nuclear positivity (Ki-67 immunostain, $\times 100)$; b: Few tumor cells showing nuclear positivity (p53 immunostain, $\times 100$ )

Table 3: Multivariate analysis of $\mathrm{Ki}-67 \mathrm{LI}$ between various grade of meningiomas

\begin{tabular}{ll}
\hline Grade I from Grade II & $P=0.000$ \\
Grade I from Grade III & $P=0.000$ \\
Grade I from recurrent cases & $P=0.010$ \\
Grade II from Grade III & $P=0.023$ \\
Grade II from recurrent cases & $P=0.034$ \\
Grade III from recurrent cases & $P=0.000$ \\
\hline
\end{tabular}


Rao, et al.: Characterization of morphologically benign biologically aggressive meningiomas

\begin{tabular}{lcc}
\hline \multicolumn{3}{l}{ Table 4: p53 expression in various grades of meningiomas } \\
\hline Grades & Range (\%) & Mean (\%) \\
\hline I & $1-75$ & 15.57 \\
II & $47-70$ & 57 \\
III & $45-77$ & 60.8 \\
Recurrent cases & $40-75$ & 62.5 \\
\hline
\end{tabular}

A total of 11 cases of grade I meningiomas showed a high Ki-67 LI and p53 expression, of which six presented with recurrence. All recurrent cases were histologically WHO grade I and showed no histological features of atypia and there was no evidence of brain invasion. However, these cases showed focal hypercellular areas. The mean Ki-67 LI and p53 expression of recurrent meningiomas were $8.6 \%$ and $62.5 \%$ respectively [Table 5]. Period of recurrence ranged between 2 and 4 years with a mean of 3.4 years. All recurrent cases showed a high Ki-67 LI and p53 expression [Figure 3]. There was one case of inadequately removed tentorial meningioma with uncal herniation who presented with regrowth of tumor. We also had five WHO grade 1 meningioma cases with high Ki-67 LI with corresponding high p53 expression and these are under follow-up for the past two years with no recurrence.

Meningiomas with good number of lymphocytes showed falsely high Ki-67 value [Figure 4], therefore hot spots with minimal lymphocyte infiltration were selected for counting in these cases to derive a true proliferative index.

\section{Discussion}

Predicting the behavior of meningiomas based on histopathologic features continues to be problematic. ${ }^{[3]}$ While tumor grade, subtype and extent of resection are useful factors, recurrence has been noted in some meningiomas with no known aggressive parameters. Aggressive meningiomas with atypical features are easily identified on histological sections and may not pose any challenge to the neuropathologist. The meningiomas of concern are those which are morphologically benign but biologically aggressive.

Several studies in the past have demonstrated a proportionate increase in proliferative index with increasing tumor grade. ${ }^{[8,4,9]}$ Similar results were also noted in our study with statistical significance in the mean value. Though assessment of proliferative activity is a good indicator of tumor aggressiveness, p53 expression was studied to evaluate its role in tumor biology. p53 is responsible for tumor progression in many malignant tumors and its over expression has been detected in these tumors. Earlier studies have demonstrated variable results with regards to alteration in p53 pathway in meningiomas ${ }^{[10-12]}$ The diverse finding of p53 expression,

\begin{tabular}{lcc}
\hline \multicolumn{3}{l}{ Table 5: Recurrent cases-Ki-67 Ll and p53 expression } \\
\hline Grade & Ki-67 LI (\%) & P53 (\%) \\
\hline Grade I & 12 & 75 \\
Grade I & 7 & 40 \\
Grade I & 7 & 65 \\
Grade I & 7 & 65 \\
Grade I & 7 & 65 \\
Grade I & 12 & 65 \\
\hline
\end{tabular}

not always correlating with tumor grade and biological aggressiveness, could depend on factors such as the number of alleles of p53 deleted and stability of p53 protein. ${ }^{[2]}$

Similar to the observations by Karamitopoulou et al., we noted a good correlation between Ki-67 LI and p53 expression. ${ }^{[2]}$ A high proliferative index and p53 expression in grade II and III meningiomas confirms their aggressive nature. A uniformly high expression of p53 in grade II and III meningiomas identifies its role in causing genomic instability in this subgroup of aggressive meningiomas.

Caution should be exercised in interpreting Ki-67 positivity, as lymphocytes or any other proliferative cells can show positivity for Ki-67 antigen. A meticulous comparison with hematoxylin and eosin section ( $\mathrm{H}$ and $\mathrm{E})$ would help in eliminating this error. This reinforces the fact that immunostains can only be used as an adjunct and cannot replace histology.

All the six recurrent cases were histologically grade I meningiomas with recurrence in spite of complete surgical resection. The recurrent menigiomas showed high proliferative activity and p53 expression. We have also noted a high Ki-67 LI and p53 expression in five of the WHO grade I meningioma cases. These patients are under follow-up for two years with no recurrence. These findings draw our attention to the fact that biological behavior of meningiomas and risk of recurrence in individual cases cannot be predicted by using conventional histological criteria alone. ${ }^{[2]}$ Recurrence may not be limited to meningiomas with aggressive histological features. Even a completely excised histologically benign meningioma may recur.

Karamitopoulou et al., found two groups of meningiomas, one group with high p53 and Ki-67 expression and another group with low values. ${ }^{[2]}$ A third unique group of morphologically benign tumors with high proliferative index and p53 expression was recognized in our study. The third group comprised 11 cases of which six presented with recurrence. A high Ki-67 LI and p53 expression in six recurrent cases emphasizes the fact that utilization of objective markers such as Ki-67 LI and p53 expression may prove useful in recognizing morphologically 

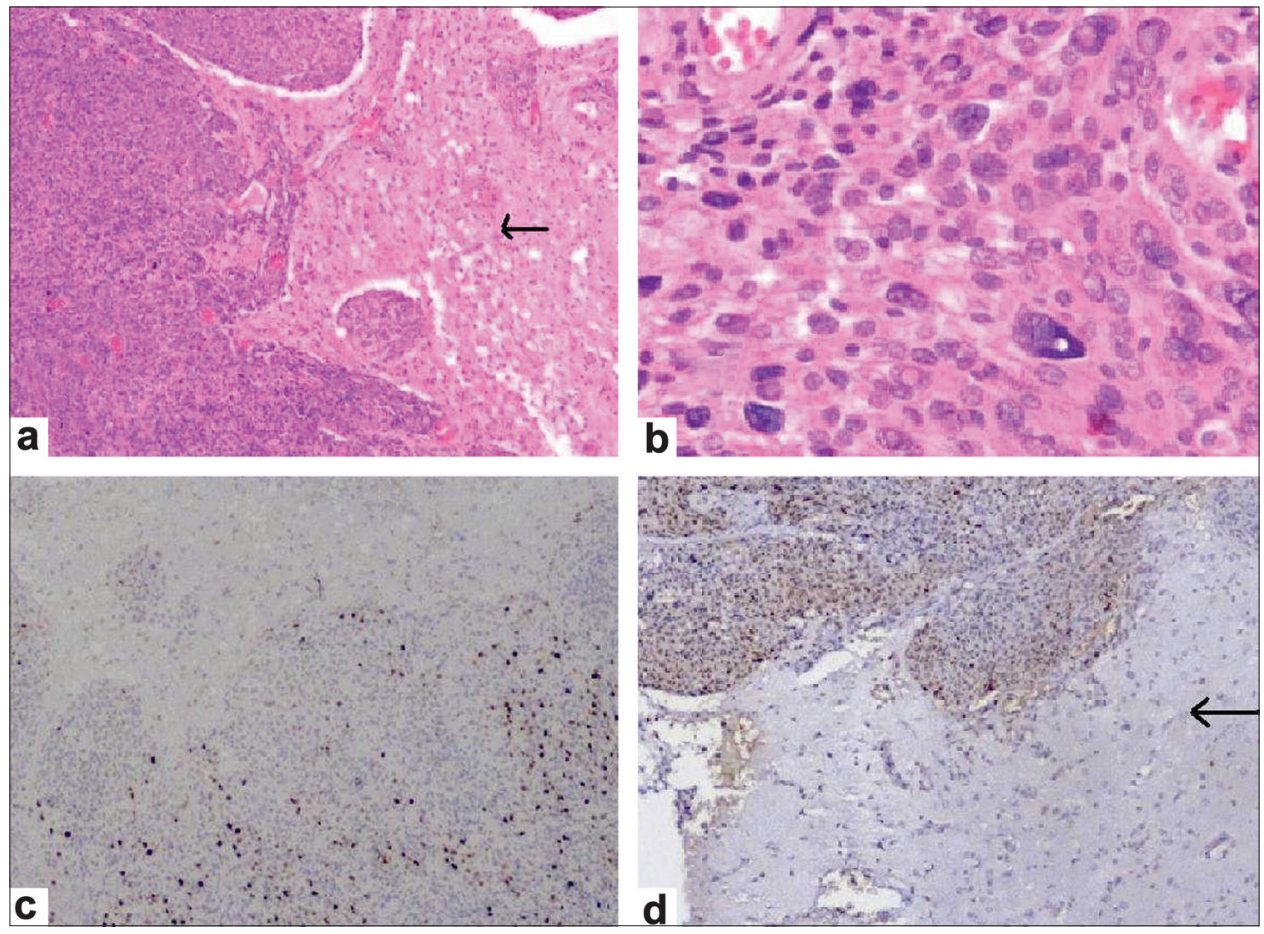

Figure 2: Sections of anaplastic meningioma-a: Cellular areas with islands of pleomorphic tumor cells infiltrating brain parenchyma (arrow) ( $H$ and $E, \times 20)$; b: Higher magnification showing highly atypical cells with increased nucleocytoplasmic ratio (H and $E, \times 100)$; $c$ : Large number of tumor cell nuclei showing positivity (Ki-67 immunostain, $\times 20$ ); $d$ : Many tumor cells with nuclear positivity invading brain tissue (arrow) (p53 immunostain, $\times 20)$
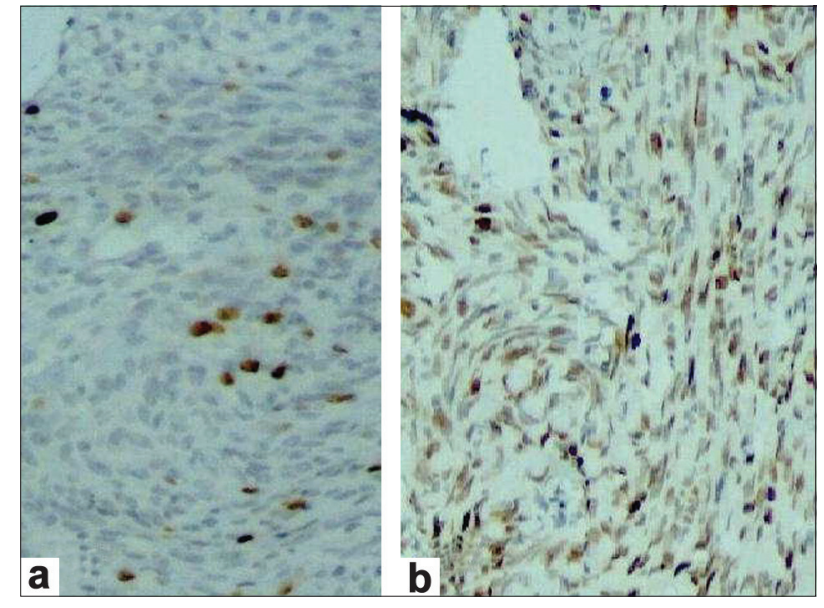

Figure 3: Sections of grade I recurrent meningiotheliomatous meningioma-a: Nuclear positivity seen in many tumor cells signifying high proliferative index (Ki-67 immunostain, $\times 100)$; b: High nuclear positivity in tumor cells (p53 immunostain, $\times 100$ )

benign meningiomas with unfavorable clinical outcome. The existence of morphologically benign, biologically aggressive meningiomas, needs to be re-emphasized since assessment of proliferative index is not a routine practice in all centres. Tyagi et al., in their analysis of MIB-I LI and other parameters suggested that extent of surgical excision and histology were two main factors influencing the prediction of recurrence and not proliferative markers. ${ }^{[13]}$ Kalala et al., in their in vitro evaluation of meningioma found no correlation between proliferative markers and the behavior in vitro. ${ }^{[14]}$ However, they demonstrated that invasion had

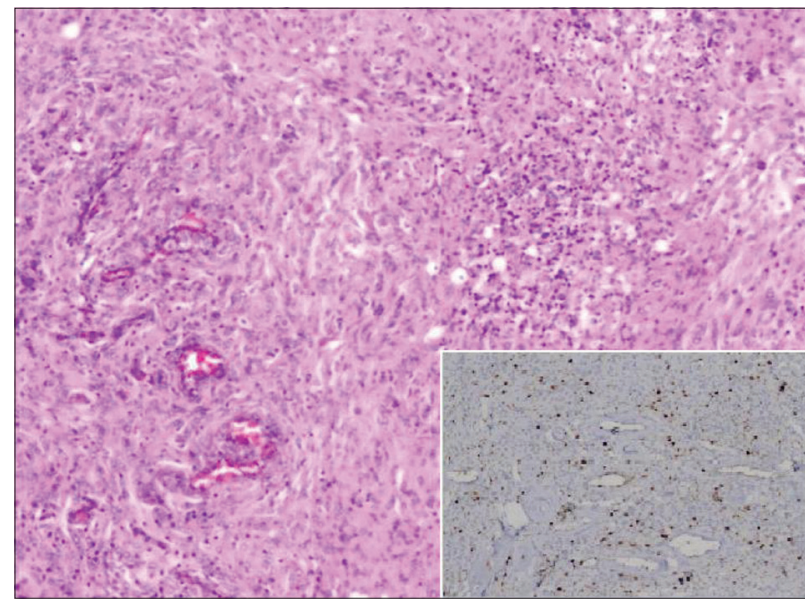

Figure 4: Meningiotheliomatous meningioma showing infiltration with a large number of lymphocytes $(\mathrm{H}$ and $\mathrm{E}, \times 40)$; inset shows positivity in the nuclei of lymphocytes but not in tumor cells (Ki-67 immunostain, $\times 20$ )

a positive correlation with recurrence and malignancy. ${ }^{[14]}$

Presently, proliferative index is not included in WHO criteria in grading meningioma and is said to be useful in tumors that are histologically "on the fence" with regards to tumor grade. ${ }^{[15]}$ An increase in both Ki-67 LI and p53 expression amongst the recurrent meningiomas and high grade meningiomas in our study, suggests that these immunostains may be of importance in identifying a subset of biologically aggressive morphologically benign meningiomas. 


\section{References}

1. Perry A, Louis DN, Scheithauer BW, Budka H, von Deimling A. Meningiomas In: Kleihues P, Cavenee WK, editors. World Health Organization Classification of Tumours of the Nervous system $3^{\text {rd }}$ edition, IARC Press: Lyon; 2007. p. 164-72.

2. Karamitopoulou E, Perentes E, Tolnay M, Probst A. Prognostic significance of MIB-1, p53 and bcl-2 Immunoreactivity in meningiomas. Hum Pathol 1998;29:140-5.

3. Abramovich CM, Prayson RA. Histopathologic features and MIB-1 labeling indices in recurrent and nonrecurrent meningiomas. Arch Pathol Lab Med 1999;123:793-800.

4. Devaprasath A, Chacko G. Diagnostic validity of the Ki-67 labeling index using the MIB-1 monoclonal antibody in the grading of meningiomas. Neurol India 2003;51:336-40.

5. Burger PC, Shibata T, Kleihues P. The use of monoclonal antibody Ki-67 in the identification of proliferating cells: Application to surgical neuropathology. Am J Surg Pathol 1986;10:611-7.

6. Cattoretti G, Becker MH, Key G, Duchrow M, Schlüter C, Galle J, et al. Monoclonal antibodies against recombinant parts Ki-67 antigen (MIB-1 and MIB-3) detect proliferating in the microwave processed formalin fixed paraffin. J Pathol 1992;168:357-63.

7. Barbareschi M, Iuzzolino P, Pennella A, Allegranza A, Arrigoni G, Dalla Palma $\mathrm{P}$, et al. p53 protein expression in central nervous system neoplasms. J Clin Pathol 1992;45:583-6.

8. Torp SH, Lindboe CF, Grønberg BH, Lydersen S, Sundstrøm S.
Prognostic significance of Ki-67/MIB-1 proliferation index in meningiomas. Clin Neuropathol 2005;24:170-4.

9. Madsen C, Schrøder HD. Ki-67 immunoreactivity in meningiomas-determination of the proliferative potential of meningiomas using the monoclonal antibody Ki-67. Clin Neuropathol 1997;16:137-42.

10. Amatya VJ, Takeshima Y, Inai K. Methylation of p14 (ARF) gene in meningiomas and its correlation to the p53 expression and mutation. Mod Pathol 2004;17:705-10.

11. Aguiar PH, Agner C, Simm R, Freitas AB, Tsanaclis AM, Plese P. p53 protein expression in meningiomas-a clinicopathologic study of 55 patients. Neurosurg Rev 2002;25:252-7.

12. Verheijen FM, Sprong M, Kloosterman JM, Blaauw G, Thijsssen JH, Blankenstein MA. TP53 mutations in human meningiomas. Int J Biol Markers 2002;17:42-8.

13. Tyagi A, Chakrabarty A, Franks A. MIB1 proliferation index in meningiomas: Does it predict recurrence? A clinicopathological study. Br J Neurosurg 2004;18:357-61.

14. Kalala JP, Benoit D, de Ridder L. Can recurrence of meningioma be predicted? Anticancer Res 2004;24:2319-24.

15. Takei H, Bhattacharjee MB, Rivera A, Dancer Y, Powell SZ. New immunohistochemical markers in the evaluation of the central nervous system tumors: A review of 7 selected adult and Pediatric brain tumors. Arch Pathol Lab Med 2007;131:234-41.

Accepted on 06-08-2009

Source of Support: Nil, Conflict of Interest: None declared. 